\title{
Система локализованных экситонов на кислородных комплексах в CdS
}

\author{
(C) Н.К. Морозова ${ }^{1}$, И.Н. Мирошникова ${ }^{1,2}$ \\ ${ }^{1}$ Национальный исследовательский университет „Московский энергетический институт“, \\ 111250 Москва, Россия \\ ${ }^{2}$ Институт нанотехнологий микроэлектроники Российской академии наук, \\ 119991 Москва, Россия \\ E-mail: MorozovaNK@mail.ru \\ Поступила в Редакцию 14 мая 2021 г. \\ В окончательной редакции 15 июня 2021 г. \\ Принята к публикации 15 июня 2021 г.
}

Интенсивное свечение $\mathrm{CdS}$ в голубой и зеленой областях спектра широко используется во всех областях оптоэлектроники. Приведены результаты исследования оптических свойств $\mathrm{CdS}$ на базе теории антипересекающихся зон с привлечением более широких исходных данных к анализу результатов. В зависимости от условий роста учтено присутствие и изменение концентрации кислорода и собственных точечных дефектов, определяющих состав кристаллов. Введены представления о неравномерном распределении изоэлектронных кислородных центров в объеме $\mathrm{CdS}$ вследствие преимущественной сегрегации их на компенсирующих дефектах упаковки. Для анализа экспериментальных данных использовались возможности построения зонных моделей, которые объединяют обширную и разностороннюю информацию о конкретных образцах. Представлена модель мультизоны CdS·O c дефектами упаковки, определяющая спектр зеленого краевого излучения. Дано объяснение природы краевого излучения сульфида кадмия как экситонов, локализованных на кислородных комплексах.

Показано, что присутствие изоэлектронных кислородных центров проявляется в электрофизических свойствах кристаллов. Даны рекомендации по диагностике кристаллов, пригодных для создания стабильных в эксплуатации люминесцирующих систем или лазеров.

Ключевые слова: изоэлектронные центры, теория антипересекающихся зон, локализованный экситон, дефекты упаковки, точечные дефекты, стимулированное излучение.

DOI: 10.21883/FTP.2021.11.51562.9650

\section{1. Введение}

Узкие полосы у края поглощения $\mathrm{CdS}$ привлекали внимание многих исследователей [1-11]. В хорошо известных работах Гросса с сотр. [2] представлены первые результаты исследований экситонов в $\mathrm{A}^{\mathrm{II}} \mathrm{B}^{\mathrm{VI}}$. Основные особенности группы полос, объединяющей компоненты зеленого краевого свечения CdS (edgeemission - EE), описаны в фундаментальной работе [1]. Для объяснения природы EE CdS была привлечена утвердившаяся в эти годы модель донорно-акцепторных (Д-А) пар [3], согласно которой акцептором мог быть дефект решетки, а различные типы примесных доноров определяли полосы краевого свечения. Различные полосы рассматривались как набор Д-А пар. Эта тенденция, как основная, сохраняется до настоящего времени [4,5]. При этом не учитывается, что лишь некоторые особенности EE CdS сходны с чертами Д-А пар, но другие им явно противоречат [1]. Все исследователи отмечали зависимость свечения от состава кристаллов [1,7-11]. Присутствие кислорода в кристаллах не учитывается, но характерно, что краевое свечение $\mathrm{CdS}$ наблюдается в образцах с избытком кадмия [1].

Развитие теоретических основ физики твердого тела и создание сравнительно недавно утвердившейся тео- рии антипересекающихся зон (bandanticrossingtheory ВАС) [6] привело к более глубокому восприятию собственно-дефектной структуры кристаллов.

\section{2. Образцы для исследования}

Ориентируясь на неизбежное присутствие кислорода и влияние собственных точечных дефектов на спектры излучения $\mathrm{CdS}$, мы исследовали реальные монокристаллы в плане возможного изменения их состава в зависимости от условий получения [11-16]. Исследования проводились на группе кристаллов, выращенных из газовой фазы в пределах области гомогенности $\mathrm{CdS}$ [12]. В соответствии с составами кристаллов рассчитано равновесие собственных точечных дефектов и определены концентрации кислорода $[\mathrm{O}]$ по данным разработанной и опробованной методики газохроматографического анализа (ХГХ) [13].

На рис. 1 представлены эти данные. Условия роста кристаллов $\mathrm{CdS}$ при $1100^{\circ} \mathrm{C}$ из газовой фазы при контролируемом давлении паров серы $(\mathrm{S})$ описаны в [11]. Расположение на рисунке штриха с номером образца определяет состав кристаллов как набор собственных точечных дефектов. Концентрация кислорода $[\mathrm{O}]$ охарактеризована длиной штрихов на рис. $1, b$. Зачернена 


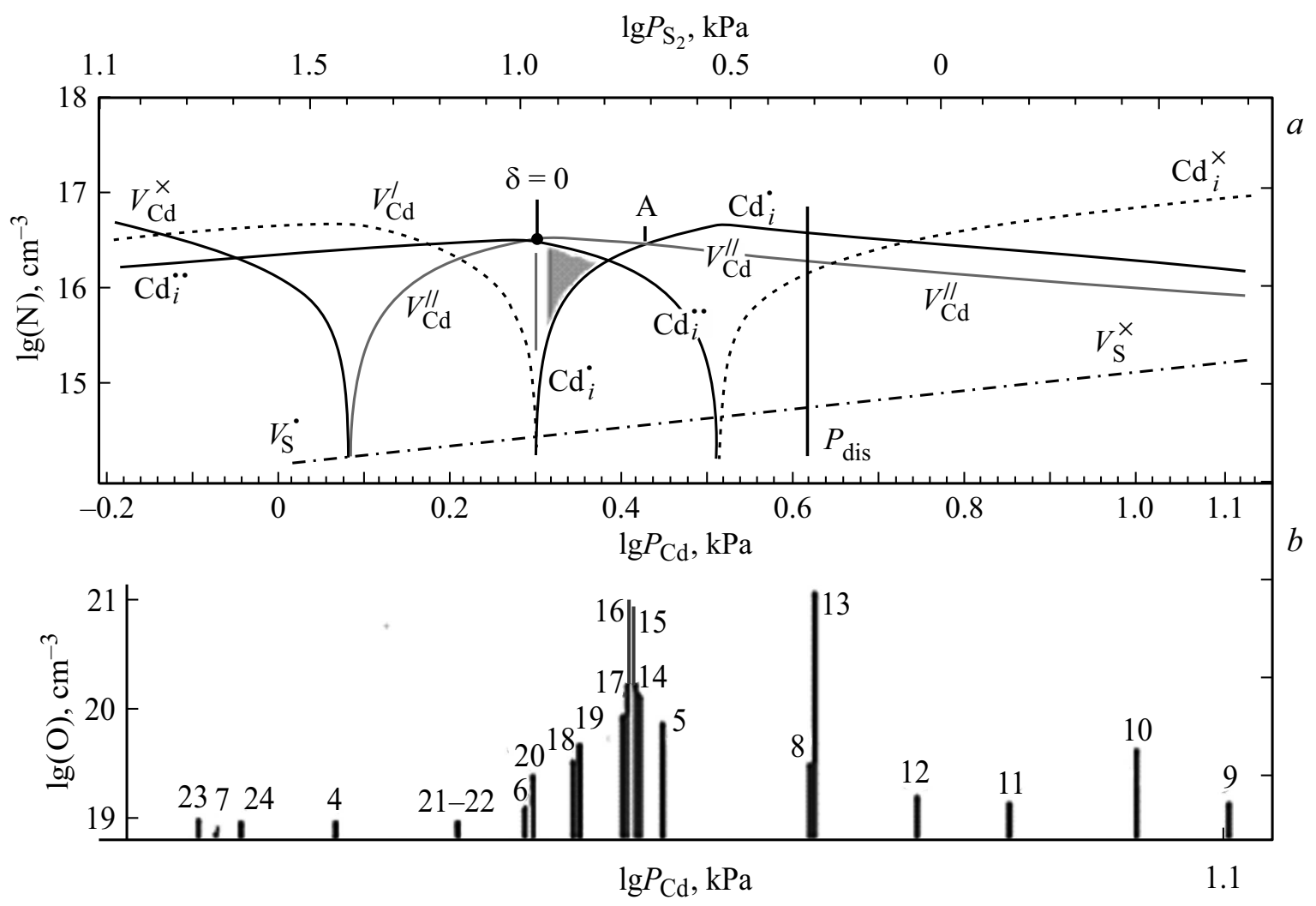

Рис. 1. Кристаллы $\mathrm{CdS}$ с указанием условий роста при $1100^{\circ} \mathrm{C}$ и содержанием в них кислорода $(b)$ в сопоставлении с расчетной диаграммой равновесия собственных точечных дефектов (СТД) (a). Зачернена „область стехиометрии“. Зарядовые состояния дефектов $0,+,++,-,--$ соответствуют обозначениям: $\times, \bullet, \bullet \bullet, /, / /[1]$.

„область стехиометрии“, которая ограничена резким спадом концентрации $\mathrm{Zn}_{i}^{\bullet}$ на диаграмме и концентрации $\mathrm{O}_{\mathrm{S}}$ в кристаллах. На эту область приходится минимум суммы общего количества дефектов согласно рис. $1, a$.

Анализ дефектообразования лег в основу изучения оптических свойств этих кристаллов с учетом зависимости их от типа собственных точечных дефектов. Кроме того, при исследованиях учитывались особенности кристаллической структуры образцов с отклонениями от стехиометрии [11].

\section{3. Спектры катодолюминесценции}

На рис. 2 представлены выборочно спектры катодолюминесценции (КЛ) группы кристаллов $\mathrm{CdS}$, выращенных в пределах области гомогенности. Спектры измерены по методике M1[11] в широком пучке при плотности возбуждения $10^{22} \mathrm{~cm}^{-3} \cdot \mathrm{c}^{-1}$ и возможности получения информации от отдельных участков облучаемого кристалла $0.5-1$ мм. Глубина информационного слоя $\sim 0.5$ мкм. Как видно из рис. 2 , наблюдаются интенсивные серии краевого свечения с головными линиями $514-516$ нм и LO мотивом. Типичный спектр представлен кристаллом № 15. Изучение всей группы кристаллов (рис. 1) показало [11], что максимальной интенсивности эти полосы достигают в „области стехиометрии“ (рис. 1, $a$ ). В работе далее приводятся данные, раскрывающие природу этих полос: их связь с концентрацией кислорода и типом СТД, зависимость от температуры и присутствия дефектов упаковки [17].

Что касается экситонной полосы 489 нм, то она для всей группы кристаллов не обнаруживает зависимости от $[\mathrm{O}]$ (рис. 2). Такая стабильность может быть связана со сравнительно постоянным составом чистых слоев в кристаллах с дефектами упаковки (ДУ). Дефекты упаковки, которые образуются для компенсации

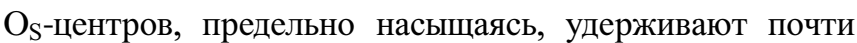
весь кислород. При этом состав светлых слоев поддерживается постоянным. Обозначим полосу 489 нм как экситон светлых слоев $\left(E_{\mathrm{ex}}^{\mathrm{cc}}\right)^{1}$.

С целью уточнения природы этой экситонной полосы $\mathrm{CdS}$ были предприняты съемки по методике M2[11] при температурах ниже азотных и при большем разрешении. На рис. 3 приведены типичные спектры монокристаллов $\mathrm{CdS}$, выращенных при различных давлениях паров компонентов.

\footnotetext{
${ }^{1}$ Положение экситонной А-полосы бескислородного $\mathrm{CdS}$ по данным $[1]-487$ нм $(80 \mathrm{~K})$. По спектральному положению полосы 489 нм светлые слои достаточно чистые по кислороду $\sim 2 \cdot 10^{19} \mathrm{~cm}^{-3}$.
} 

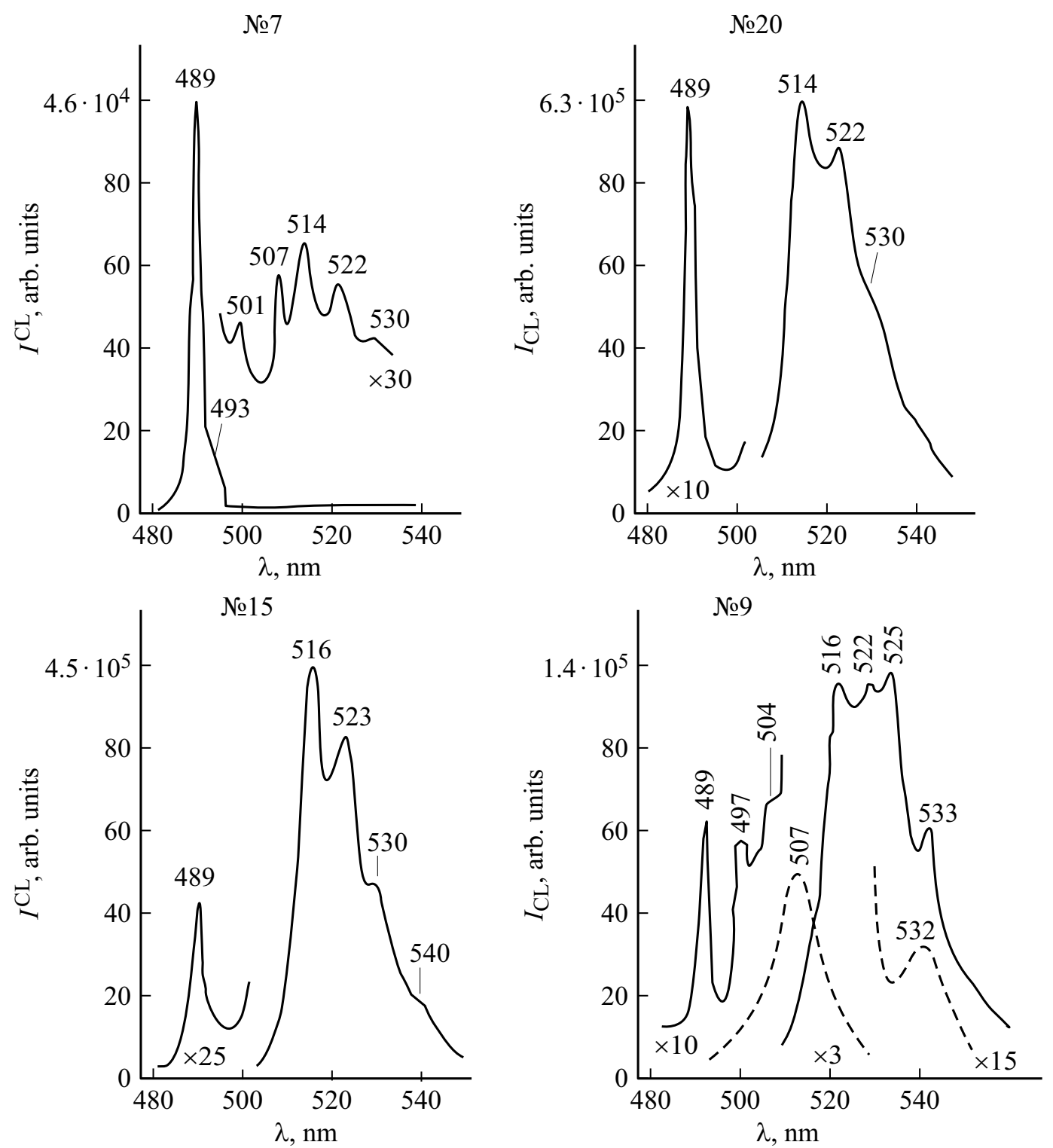

Рис. 2. Спектры КЛ, измеренные по методике М1 при $80 \mathrm{~K}$ (пунктир $-300 \mathrm{~K}$ ). Плотность возбуждения $G=10^{22} \mathrm{~cm}^{-3} \cdot \mathrm{c}^{-1}$.

Как видно из рис. 3, в экситонной области спектра разрешаются две полосы 489 и 487 нм. Изучение спектров всей группы кристаллов показало, что каждая из полос 489 и 487 нм сопровождается различным „краевым“ свечением и различно зависит от состава кристаллов и содержания кислорода.

Экситонная полоса 487 нм наблюдается в спектрах чистых по кислороду образцов, выращенных при избыточных давлениях паров серы.

В краевой области спектра таких кристаллов при $47 \mathrm{~K}$ мы наблюдали единственную серию полос с головной линией 507 нм и LO повторами (см. вставку на рис. 3). Эта наиболее коротковолновая (КВ) серия в области краевого спектра наблюдалась только при $47 \mathrm{~K}$ как самостоятельная при минимальном количестве кислорода $8.7 \cdot 10^{18} \mathrm{~cm}^{-3}$ в образце № 7 .
Этот тип свечения детально описан в работах $[11,14,16,18]$ для $\mathrm{CdS} \mathrm{ZnS}$ и $\mathrm{ZnSe}$ как $\mathrm{SAL}^{2}$. Свечение наблюдается при низких температурах для составов, близких к чистому по кислороду кристаллу.

\footnotetext{
${ }^{2}$ В этих работах введены обозначения двух видов разного по природе самоактивированного свечения $\mathrm{A}^{\mathrm{II}} \mathrm{B}^{\mathrm{VI}}$. Это SA-свечение на глубоких уровнях рекомбинации для $\mathrm{CdS} \sim 0.8$ эВ, обязанных комплексам $\left\{\mathrm{O}_{\mathrm{S}} \mathrm{Cd}_{i}^{\bullet} V_{\mathrm{Cd}}^{/ /}\right\}$. Оно реализуется для составов кристаллов, содержащих в большой концентрации составляющие комплекса, в частности $\mathrm{Cd}_{i}^{\bullet}$ и $\mathrm{O}_{\mathrm{S}}$. На рис. $1, a$ это составы от границы „области стехиометрии“ к избытку кадмия.

SAL-свечение реализуется с участием других комплексов собственных точечных дефектов $\left\{\mathrm{Cd}_{i}^{\bullet \bullet} V_{\mathrm{Cd}}^{/ /}\right\}^{\times}$, которые определяют мелкие акцепторные уровни, для $\mathrm{CdS} \sim 0.12$ эВ при $80 \mathrm{~K}$ Свечение наблюдается для составов за пределами „области стехиометрии“, близких к точке $\delta=0$ на рис. $1, a$. Возникновение SAL-центров соответствует низким концентрациям кислорода.
} 

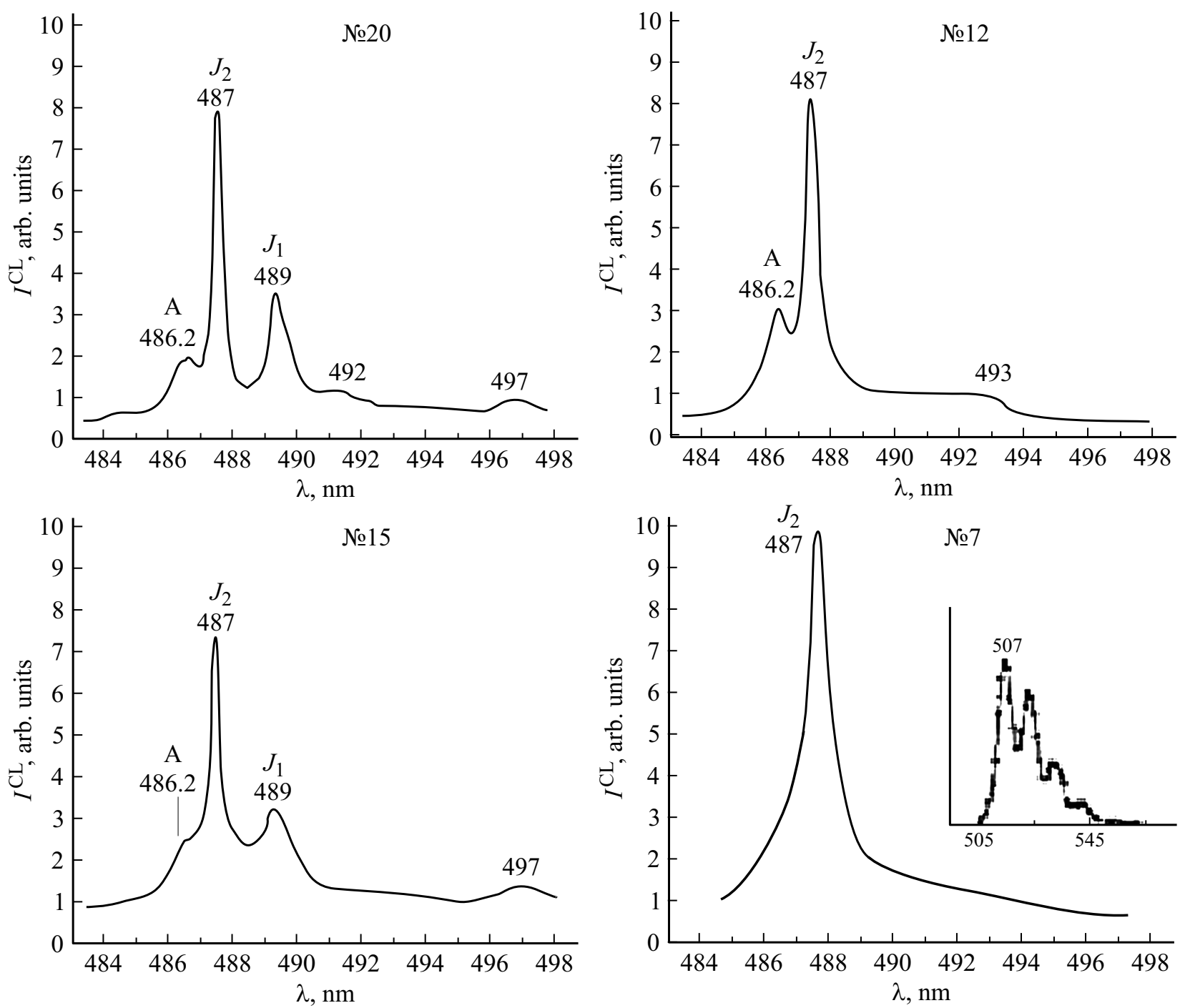

Рис. 3. Спектры КЛ, снятые по методике $\mathrm{M} 2^{3}$ при $47 \mathrm{~K}$ и плотности возбуждения $10^{21} \mathrm{~cm}^{-3} \cdot \mathrm{c}^{-1}$. На вставке приведена серия краевого свечения локальной области неоднородного кристалла № 7.

Что касается экситонной полосы 489 нм, то в спектрах чистых по кислороду кристаллов она не наблюдается (рис. 3, образцы № 7,12). При $80 \mathrm{~K}$ во всех случаях эта полоса 489 нм в спектрах рис. 2 сопровождалась линиями краевого свечения $\mathrm{CdS} 514,515,516$ нм, которые с понижением температуры измерения до $47 \mathrm{~K}$ не обнаруживаются (рис. 3), даже если наблюдается полоса 489 нм. Тем не менее полосу 489 нм мы связываем с дефектами упаковки, и, как показано далее, можно считать, что обнаружение ее является признаком присутствия ДУ в кристалле.

Отметим также особенности спектров, выявленные при исследовании всей группы кристаллов локальной съемкой. Для отдельных участков неоднородных кристаллов, например образец № 10 , наряду $E_{\mathrm{ex}}^{\mathrm{cc}} 489 \mathrm{Hм}$ возникает и усиливается полоса 497 нм - при этом компоненты краевого свечения уменышаются вплоть до уровня фона.
Полоса 497 нм выделяется и как индивидуальная (рис. 4).

Ей в спектрах отражения сопутствует резко выраженный край дополнительного поглощения 496 нм (рис. 4, кривая 2), ограниченный с длинноволновой стороны селективной полосой 495-497 нм полушириной 9-20 мэВ. Концентрация кислорода $\left[\mathrm{O}_{\mathrm{S}}\right]$ по положению экситонной полосы $-1.13 \cdot 10^{20} \mathrm{~cm}^{-3}$ приближается к уровню предельного легирования ДУ и результатам ХГХ анализа.

Спектр свидетельствует о повышенном содержании [13] растворенного кислорода в объеме кристалла. При уменьшении ширины запрещенной зоны основы вследствие предельного легирования кислородом почти все компоненты краевого свечения перекрываются с

\footnotetext{
3 Гелиевый криостат позволял достигать температуры $28-30 \mathrm{~K}$ уровень возбуждения $10^{22} \mathrm{~cm}^{-3} \cdot \mathrm{c}^{-1}$. Глубина информационного слоя $\sim 0.5$ мкм. Дифракционная решетка обеспечивала дисперсию $7.4 \AA / \mathrm{Mм}$.
} 

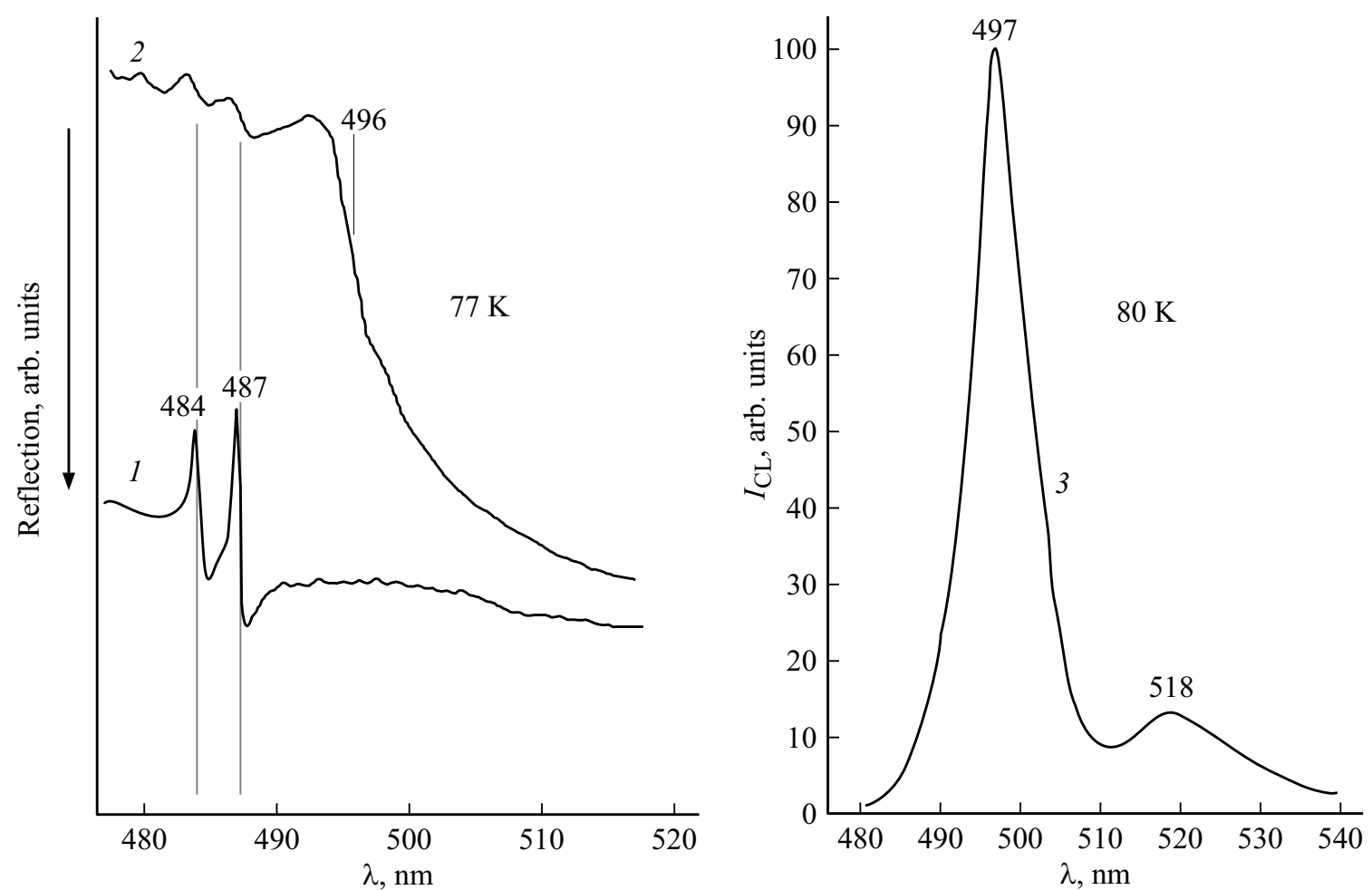

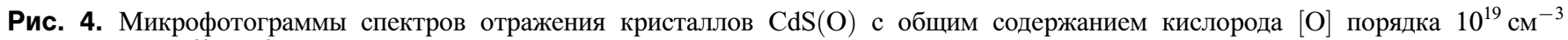
(кривая 1) и $10^{20} \mathrm{~cm}^{-3}$ (кривая 2), а также спектр КЛ кристалла № 18 из области кислородных скоплений (кривая 3).

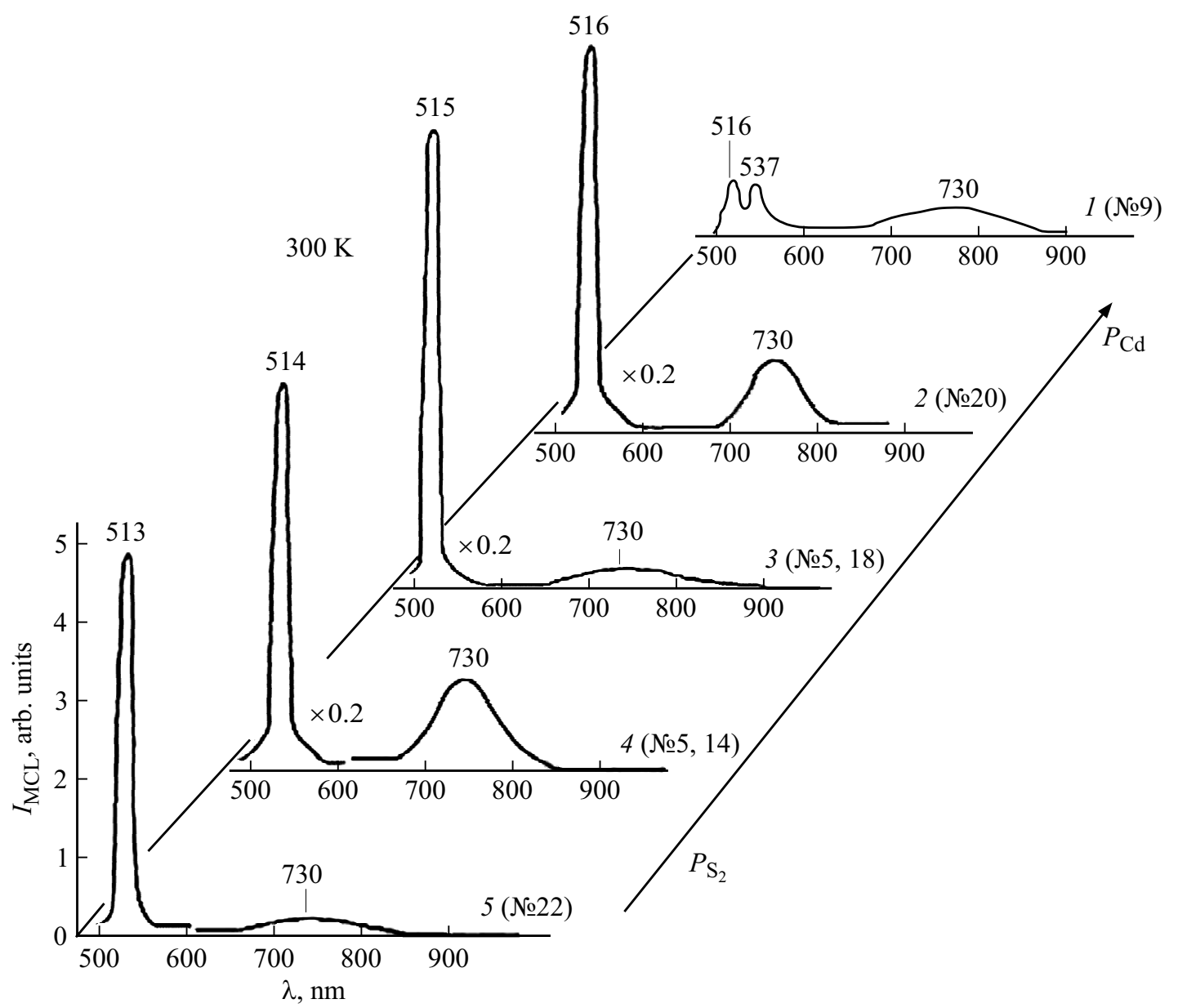

Рис. 5. Спектры МКЛ сняты в РЭМ при $300 \mathrm{~K}$, энергии пучка 25 кэВ и плотности возбуждения $10^{24} \mathrm{~cm}^{-3} \cdot \mathrm{c}^{-1}$. 
полосой 497 нм. Для образца № 18 (рис. 4) разрешается только наиболее длинноволновая (ДВ) компонента - 518 нм. Экситонная полоса 497 нм, таким образом, косвенно свидетельствует о ширине запрещенной зоны и уровне предельного легирования $\mathrm{CdS}$ кислородом.

\section{4. Микрокатодолюминесценция}

Чтобы исключить влияние неоднородностей структуры при изучении зависимости оптических свойств кристаллов $\mathrm{CdS}$ от состава, были предприняты исследования спектров микрокатодолюминесценции (МКЛ) в растровом электронном микроскопе (РЭМ) при $300 \mathrm{~K}$ и большей интенсивности возбуждения. Спектры МКЛ снимались от отдельных моноблоков при увеличении $\times 10000$ и размере растра чаще всего $10 \times 10$ мкм (диаметр пучка $1000 \AA$ ).

Спектры приведены на рис. 5 выборочно для кристаллов, выращенных в пределах области гомогенности $\mathrm{CdS}$ (рис. $1, b$ ). Как видно, при повышении температуры съемки до $300 \mathrm{~K}$ в спектрах наблюдаются полосы зеленого краевого свечения на тех же кристаллах и при тех же длинах волн 513, 514, 515, 516 нм.

На рис. 5 приведены результаты, соответствующие съемке центральной части моноблоков ${ }^{4}$. В целом выявляется тенденция высокоэнергетического сдвига максимума полос с составом кристаллов - увеличением $P_{\mathrm{S} 2}$ при росте. Диапазон смещения по всем образцам в пределах от 518 до 511 нм.

\section{5. Модель зеленого краевого свечения}

В работе [19] для кристаллов $\mathrm{ZnS}$ - О с ДУ (stacking faults) было показано, что полосы в области краевого свечения являются экситонными компонентами спектра

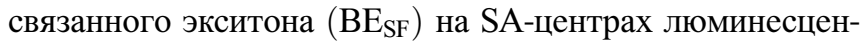
ции в слоях дефектов упаковки.

Особенности ,зеленого краевого свечения $\mathrm{CdS}^{\text {“ } ~ п р и ~}$ температуре $\sim 20 \mathrm{~K}$, соответствующие нашим результатам, описаны в основополагающей работе [1]. Для объяснения их природы была привлечена утвердившаяся в те годы модель донорно-акцепторных (Д-А) пар, согласно которой акцептором мог быть дефект решетки, а различные типы примесных доноров определяли полосы краевого свечения. Различные полосы рассматривались как набор Д-А пар. Эта тенденция, как основная, сохраняется до настоящего времени [4,5]. При этом не учитывается, что лишь некоторые особенности краевого свечения $\mathrm{CdS}$ сходны с чертами Д-А пар, но другие им явно противоречат [1]. Присутствие кислорода в

\footnotetext{
4 Полоса 537 нм, обязанная экситонному свечению $\mathrm{CdO}$ в неоднородных кристаллах, и 730 нм - SA(H) компонента самоактивированного излучения $\mathrm{CdS}$ здесь не описываются $[11,16,18]$.
}

Сравнение расчетных $\left[\mathrm{O}_{\mathrm{S}}\right]$ при $G=80$ мэВ и данные ХГХ анализов $[\mathrm{O}]$ в исследованных кристаллах $\mathrm{CdS}$

\begin{tabular}{c|c|c|c}
\hline $\begin{array}{c}\text { № } \\
\text { кристалла }\end{array}$ & $\begin{array}{c}\text { Полоса } \mathrm{BE} \\
\text { нм }(э \mathrm{SF})\end{array}$ & $\begin{array}{c}{\left[\mathrm{O}_{\mathrm{S}}\right], \mathrm{cm}^{-3}} \\
\text { расчет по } \Delta\end{array}$ & $\begin{array}{c}{[\mathrm{O}], \mathrm{cm}^{-3}} \\
\text { данные ХГХ }\end{array}$ \\
\hline $9,13,8,17,19$ & $516(2.413)$ & $1.8 \cdot 10^{20}$ & $1.2 \cdot 10^{20}$ \\
18 & $515(2.407)$ & $1.7 \cdot 10^{20}$ & $1.2 \cdot 10^{20}$ \\
14 & $514(2.412)$ & $1.6 \cdot 10^{20}$ & $1 \cdot 10^{20}$ \\
5 & $513(2.417)$ & $1.49 \cdot 10^{20}$ & $8 \cdot 10^{19}$ \\
20 & $512(2.422)$ & $7 \cdot 10^{19}$ & $3 \cdot 10^{19}$
\end{tabular}

кристаллах не учитывалось, но характерно то, что краевое свечение $\mathrm{CdS}$ наблюдается в образцах с избытком кадмия [1].

Согласно принятой нами методике анализа на основе теории ВАC [9-12], для спектра зеленого краевого свечения была построена модель мультизоны $\mathrm{CdS} \cdot \mathrm{O}$ с ДУ. Модель приведена на рис. 6, $b$ для $\mathrm{CdS} \cdot \mathrm{O}$ с ДУ.

Левая часть диаграммы $(a)$ соответствует чистым кристаллам CdS · O c [O] порядка 0.1 мол\% [11]. Для этих составов, согласно рис. 1, $a$, уменьшается концентрация дефектов $\mathrm{Cd}_{i}^{\bullet}$ почти до нуля и, следовательно, невозможно образование SA-центров и ДУ $[18,19]$.

Правая часть диаграммы $(b)$ соответствует образцам $\mathrm{CdS} \cdot \mathrm{O}$ с ДУ. Светлые слои по положению экситонной полосы $489 \mathrm{HM}\left(2.535\right.$ эВ) при $80 \mathrm{~K}$ или $E_{\mathrm{ex}}^{\mathrm{cc}}$ соответствуют описанным выше спектрам КЛ с $\left[\mathrm{O}_{\mathrm{S}}\right] \sim 2 \cdot 10^{19} \mathrm{~cm}^{-3}$.

Как уже отмечалось, полосы краевого свечения $\mathrm{CdS} \cdot \mathrm{O}$ с ДУ обусловлены экситонами $\mathrm{BE}_{\mathrm{SF}}$ на SA-центрах в слоях дефектов упаковки $[18,19]$. Для отдельных полос краевого свечения, уровни связанного экситона $\left(E_{-}^{\mathrm{BE}}\right)$ построены на рис. $6, b$ в соответствии с общей моделью излучательных экситонных переходов при энергии связи 80 мэВ. Как усреднение для всех близко расположенных уровней $E_{-}^{\mathrm{BE}}$, введем обозначение дна подзоны слоя дефекта упаковки $E$-(SF).

Модель рис. 6 определяет величины $\Delta=E_{-}^{\mathrm{cc}}-E_{-}^{\mathrm{BE}}$. Эти данные занесены в таблицу. Результаты хорошо согласуются с измеренными по спектрам КЛ (М2) расстояниями между экситонными полосами светлых слоев и полос $\mathrm{BE}_{\mathrm{SF}}$. В спектрах исследованных нами кристаллов эти величины порядка 120-140 мэВ.

Концентрация кислорода $\left[\mathrm{O}_{\mathrm{S}}\right]$, рассчитанная по величине $\Delta$ и соответствующая спектральному положению конкретной полосы связанного экситона $\mathrm{BE}_{\mathrm{SF}}$, как и анализы ХГХ [O], также приведена в таблице.

Расчетные концентрации кислорода по величине $\Delta$ для группы исследованных нами кристаллов с краевым свечением в диапазоне 513-518 нм составляют $(1.5-1.8) \cdot 10^{20} \mathrm{~cm}^{-3}$. Эти данные $\left[\mathrm{O}_{\mathrm{S}}\right]$ симбатны результатам ХГХ анализа, но превышают последние вследствие того, что при наличии дефектов упаковки ХГХ анализ дает содержание кислорода [O] на единицу объема кристалла, т.е. усредняет кислород на ДУ и в чистых слоях. 

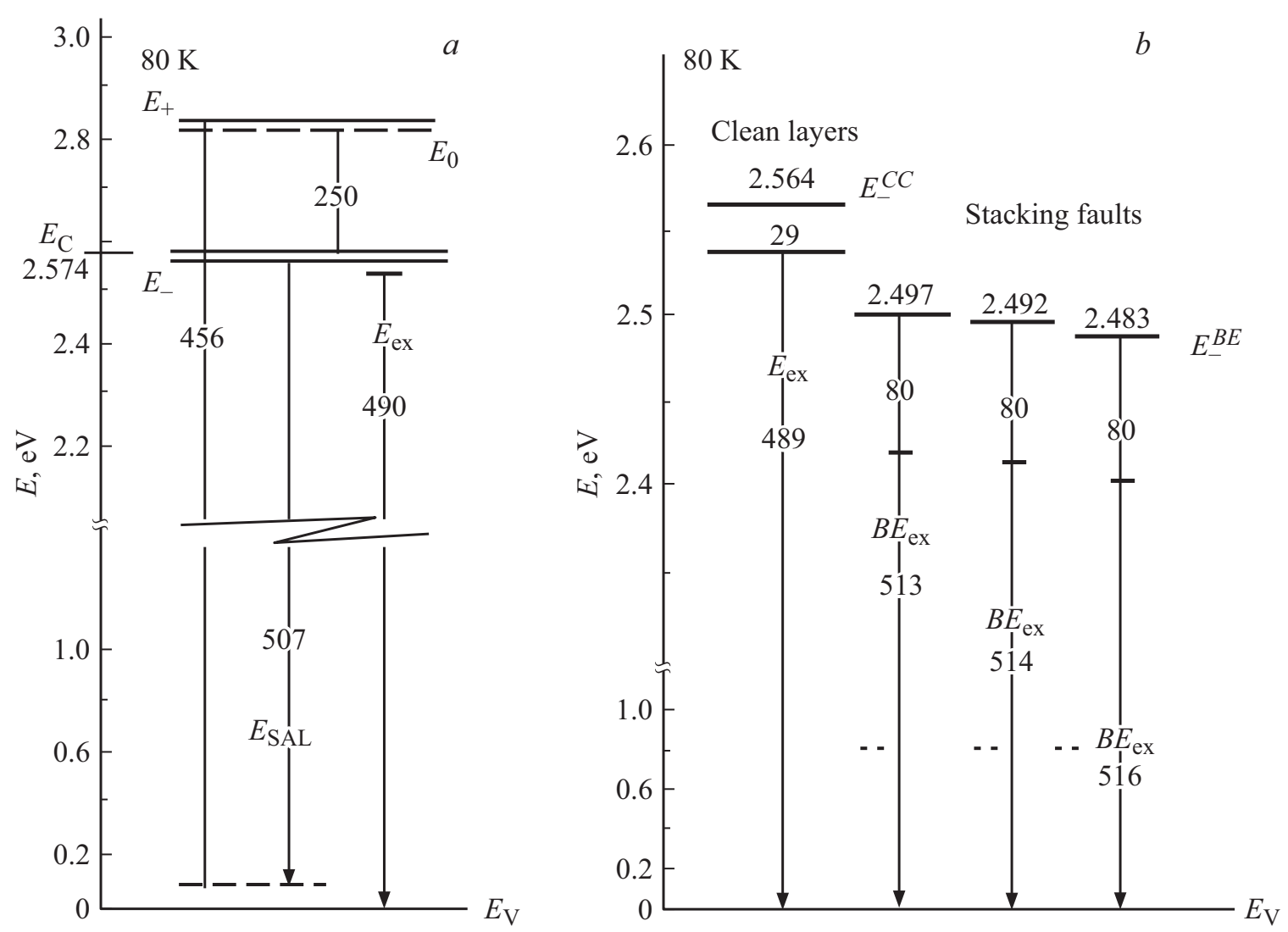

Рис. 6. Зонные модели кристаллов $\mathrm{CdS} \cdot \mathrm{O}$ : чистого без ДУ $(a)$, модель мультизоны для кристалла с ДУ $(b)$. Энергия связи $\mathrm{G}$ связанного экситона для гексагонального $\mathrm{CdS} \cdot \mathrm{O}$ взята равной 80 мэВ в соответствии с полученной в [17] для вюрцита. Пунктиром намечены глубокие уровни рекомбинации $E_{\mathrm{SA}}$, самоактивированного SA-излучения, как и при $80 \mathrm{~K}$ (рис.2).

Рассчитанные по спектрам зеленого краевого свечения $\mathrm{CdS} \cdot \mathrm{O}$ величины концентраций кислорода на ДУ выявляют сравнительно неширокий диапазон составов кристаллов, который соответствует области с максимальной интенсивностью SA-свечения и предельной растворимостью кислорода в $\mathrm{CdS}$ (область точки А на диаграмме рис. $1, a)$. Для этой области составов кислород полностью компенсирован собственными точечными дефектами решетки [20,21].

Система уровней локализованных экситонов на ДУ, как следует из анализа описанных выше спектров КЛ, не изменяется с температурой. Полосы $\mathrm{BE}_{\mathrm{SF}}$ наблюдались при одних и тех же длинах волн как при 80, так и при $300 \mathrm{~K}$.

При изменении в определенных пределах концентрации $\left[\mathrm{O}_{\mathrm{S}}\right]$ в кристалле сохраняется спектр, характерный для ДУ. Он включает полосы светлых слоев с равновесной концентрацией растворенного кислорода и сопровождающие полосы краевого свечения $\mathrm{BE}_{\mathrm{SF}}$ в слоях ДУ. При этом экситонная полоса светлых слоев $E_{\mathrm{ex}}^{\mathrm{cc}}$ наблюдалась при низких температурах измерения, когда ширина запрещенной зоны основы больше и уровень основы $E_{-}^{\mathrm{cc}}$ выше, чем $E_{-}^{\mathrm{BE}}$ в слоях дефектов упаковки. При $300 \mathrm{~K}$ соотношение меняется, и излучение может определяться отдельными полосами краевого свечения $\mathrm{BE}_{\mathrm{SF}}$.
Подобная ситуация наблюдалась в спектрах МКЛ предельно насыщенных кислородом кристаллов CdS · O, подвергнутых ионному легированию $[18,20]^{5}$. При отжиге их с уменьшением дефектности наблюдалось существенное усиление полос экситонного спектра. Выведение кислорода и изменение состава слоев при отжиге сопровождалось КВ смещением экситонной полосы основы $E_{\mathrm{ex}}^{\mathrm{cc}}$. При этом экситонная полоса основы наблюдалась только при $100 \mathrm{~K}$, например 495-494 нм. При $300 \mathrm{~K}$ в экситонной области тех же образцов вместо нее превалируют полосы $\mathrm{BE}_{\mathrm{SF}}$ краевого свечения, например $513 \rightarrow 512$ нм. Спектральное положение этих полос $\mathrm{BE}_{\mathrm{SF}}$ и экситона основы $E_{\mathrm{ex}}^{\mathrm{cc}}$ позволяет, согласно таблице, определить $\left[\mathrm{O}_{\mathrm{S}}\right]$ соответственно на ДУ и в основе. Хорошее совпадение экспериментальных и расчетных результатов подтверждает дееспособность представленной модели.

Дальнейшее изменение состава кристалла при отжиге с переходом к избытку серы показало, что уже для положения полосы $\mathrm{BE}_{\mathrm{SF}} 512$ нм концентрация кислорода $\left[\mathrm{O}_{\mathrm{S}}\right]$ выравнивается до уровня $\leq 10^{19} \mathrm{~cm}^{-3}$ во всем объеме кристалла без образования ДУ.

\footnotetext{
${ }^{5}$ Ионная имплантация до $\sim 4 \cdot 10^{20} \mathrm{~cm}^{-3}$ приводила к смещению экситонной полосы МКЛ основы до 524-525 нм при $100 \mathrm{~K}$.
} 

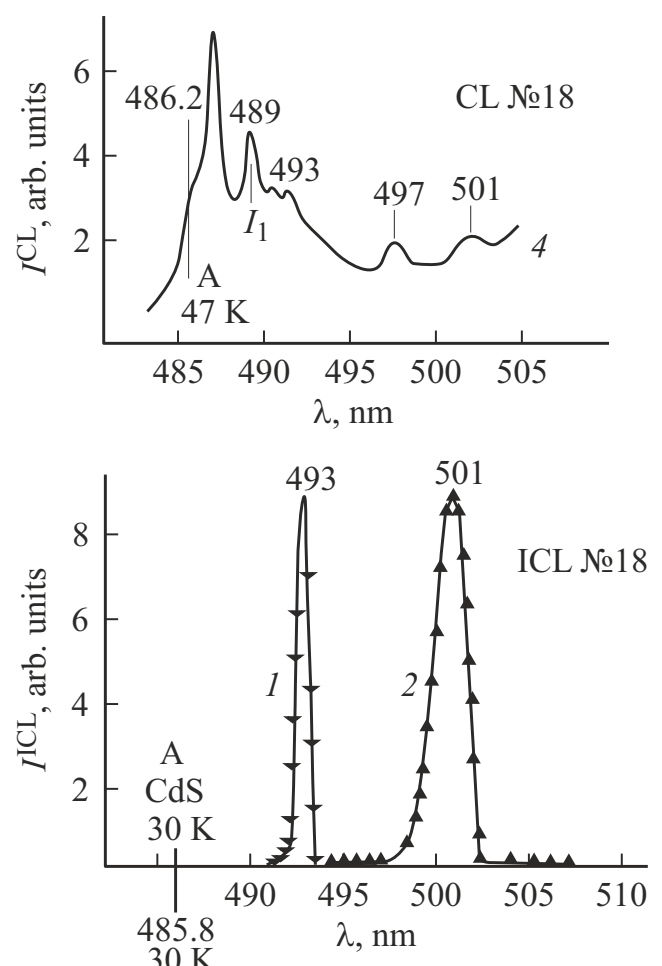

$30 \mathrm{~K}$

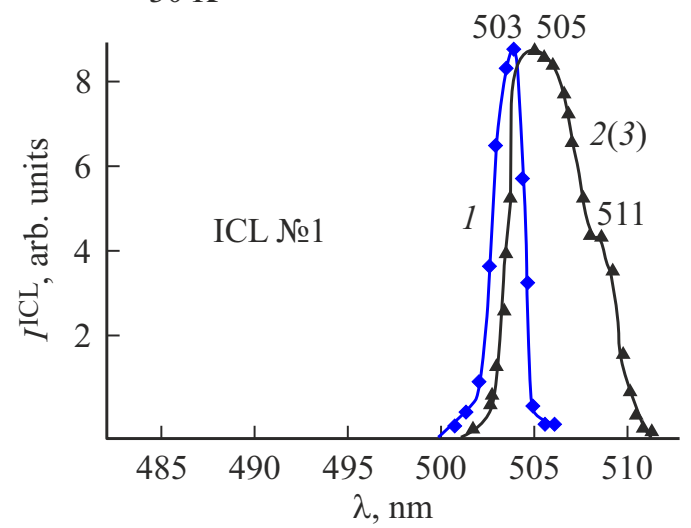

Рис. 7. Спектры люминесценции $\mathrm{CdS}(\mathrm{O})$ измерены при $47 \mathrm{~K}$ - КЛ и при $30 \mathrm{~K}$ - ИКЛ. Плотность возбуждения составляла: КЛ $\sim 10^{21} \mathrm{~cm}^{-3} \cdot \mathrm{c}^{-1}(4)$; ИКЛ $-1.56 \cdot 10^{26}(1)$; $6.52 \cdot 10^{26}(2)$ и $7 \cdot 10^{26} \mathrm{~cm}^{-3} \cdot \mathrm{c}^{-1}(3)$.

\section{6. Импульсная катодолюминесценция}

Рассмотрим изменение спектров КЛ кристаллов с ДУ при возбуждении сильноточным электронным пучком - импульсную катодолюминесценцию (ИКЛ). Измерения ИКЛ проводились при энергии электронного пучка $0.1-0.02$ Дж/см², соответствующей уровням возбуждения $\left(10^{26}-10^{27}\right) \mathrm{cm}^{-3} \cdot \mathrm{c}^{-1}$ на установке Томского НИТПУ. Глубина проникновения пучка электронов в объем образца составляла 125-200 мкм, диаметр пучка 5-10 мм [20,22].

На рис. 7 представлены спектры ИКЛ кристалла № 18 (кривые $1 \rightarrow 2$ ) стехиометрического состава с содержанием кислорода $[\mathrm{O}]=5 \cdot 10^{19} \mathrm{~cm}^{-3}$. Спектры
ИКЛ приводятся в сравнении со спектром КЛ спонтанного (экситонного) излучения этого же кристалла № 18 (кривая 4), чтобы установить связь между ними. Рассмотрим экситонную полосу 489 нм, описанную выше как $E_{\mathrm{ex}}^{\mathrm{cc}}$ слоев. Она широко известна в литературе как $I_{1}[16-18]$. При увеличении уровня возбуждения электронным пучком в спектрах ИКЛ наблюдается перестройка спонтанного излучения с увеличением интенсивности и полуширины этой экситонной полосы.

При плотности возбуждения $\leq 1.56 \cdot 10^{26} \mathrm{~cm}^{-3} \cdot \mathrm{c}^{-1}$ в спектрах ИКЛ образца № 18 возникает узкая полоса стимулированного излучения 493 нм (рис. 7, кривая 1) в области первого фононного повтора экситонной полосы светлых слоев 489 нм $E_{\mathrm{ex}}^{\mathrm{cc}}$. Достигается режим генерации. Согласно [22] генерация (лазерное излучение) на полосе 493 нм наблюдается на свободных экситонах, взаимодействующих с оптическими фононами.

С увеличением уровня возбуждения кристалла № 18 до $6.52 \cdot 10^{26} \mathrm{~cm}^{-3} \cdot \mathrm{c}^{-1}$ полоса 493 нм усиливается, уширяется и смещается в длинноволновую область до положения $501 \mathrm{Hм}$, близкого ко второму фононному повтору $E_{\mathrm{ex}}^{\mathrm{cc}}$ (рис. 7, кривая 2).

Для выяснения связи с концентрацией $\left[\mathrm{O}_{\mathrm{S}}\right]$ полос ИКЛ на рис. 7 приведен спектр кристалла $\mathrm{CdS}$ № 1 стехиометрического состава, выращенного из расплава, когда кислород мог быть введен в большей концентрации $\left(1.2 \cdot 10^{20} \mathrm{~cm}^{-3}\right)$ по сравнению с рассмотренным выше кристаллом № 18.

Как видно из рис. 7, для кристалла № 1 при той же плотности возбуждения $1.56 \cdot 10^{26} \mathrm{~cm}^{-3} \cdot \mathrm{c}^{-1}$ наблюдалась генерация, как и для образца № 18, но стимулированное излучение соответствует - 503 нм первому фононному повтору A-LO уже другой экситонной полосы 497 нм. Как мы отмечали выше, спектральное положение полосы 497 нм выявляет кристалл с предельной концентрацией растворенного кислорода в объеме CdS. Надо полагать, что в случае кристалла № 1 возбуждается объем сильно легированного кислородом образца ${ }^{6}$.

Согласно спектральному положению экситонной полосы 497 нм, концентрация кислорода в кристалле № 1 совпадает с результатами ХГХ анализа $[\mathrm{O}] \sim 1.2 \cdot 10^{20} \mathrm{~cm}^{-3}$, что соответствует и уровню легирования кислородом дефектов упаковки.

При увеличении возбуждения кристалла № 1 до $6.52 \cdot 10^{26} \mathrm{~cm}^{-3} \cdot \mathrm{c}^{-1}$ полоса 503 нм сдвигается до 505 нм. В максимуме 505 нм наблюдалось усиление - суперлюминесценция. Это подтверждается резким увеличением интенсивности свечения и появлением направленности [22]. Усиление происходит на уровнях тех дефектов,

\footnotetext{
${ }^{6}$ Предельное легирование кислородом предполагает для теоретически однородного кристалла совпадение уровня $E_{\mathrm{C}}$ основы и уровня наиболее ДВ компонент спектра краевого свечения. Для реальных кристаллов, сильно легированных кислородом, при высокой плотности возбуждения в спектрах ИКЛ наблюдались полосы $\mathrm{BE}_{\mathrm{SF}}$, но самые длинноволновые. Так, в спектре кристалла № 1 при $15 \mathrm{~K}$ - это полосы 517 и 519 нм [5], а для кристалла № 18 при $80 \mathrm{~K}$ - полоса 518 нм (рис. 4).
} 
которые ответственны за полосу спонтанной люминесценции 497 нм, т. е. для образца $\mathrm{CdS}$ № 1 определяется излучением экситона объема твердого раствора $\mathrm{CdS}(\mathrm{O})$, предельно насыщенного кислородом.

Стабилизация спектрального положения, интенсивности и формы полосы стимулированного излучения из объема образца $\mathrm{CdS}$ № 1 (кривая 3) происходит, но этот процесс наблюдается при более высоких уровнях возбуждения.

На основании рассмотренных выше результатов можно ожидать, что минимальные пороги генерации и максимальная эффективность CdS-лазеров с электронной накачкой при 80-300 K [22] может быть достигнута на образцах сульфида кадмия с ДУ состава „области стехиометрии“, т.е. с меньшим содержанием кислорода. При этом за генерацию ответственны чистые слои.

\section{Финансирование работы}

Работа выполнена при финансовой поддержке программы гранта РФФИ № 18-29-11051.

\section{Конфликт интересов}

Авторы заявляют, что у них нет конфликта интересов.

\section{Список литературы}

[1] Физика и химия соединений $\mathrm{A}^{\mathrm{II}} \mathrm{B}^{\mathrm{IV}}$, под ред. М. Авен, Д. Пренер (М., Мир, 1970).

[2] Е.Ф. Гросс, Б.С. Разбирин, С.А. Пермогоров. ФТТ, 7, 558 (1965).

[3] D.G. Thomas, M. Gershenson, F.A. Trumbore. Phys. Rev., 133, A269 (1964).

[4] А.Л. Гурский, Е.В. Луценко, Н.К. Морозова, Г.П. Яблонский. ФТТ, 34 (11), 3530 (1992).

[5] V.I. Oleshko, S.S. Vilchinskaya, V.M. Lisisyn, V.I. Korepanov. Funct. Mater., 18 (4), 457 (2011).

[6] W. Walukiewicz, W. Shan, K.M. Yu, M.J. Seong, H. Alawadhi, A.K. Ramdas. Phys. Rev. Lett., 85 (7), 1552 (2000).

[7] Н.А. Власенко, Н.И. Витриховский, З.Л. Денисова. Опт. и спектр., 21 (4), 466 (1966).

[8] И.Б. Ермолович, А.В. Любченко, М.К. Шейнкман. ФТП, 2 (11), 1639 (1968).

[9] Г.В. Бушуева, В.И. Решетов, А.А. Хромов, С.А. Пендюр, С.А. Носибов, А.Н. Печенов. ФТП, 22 (2), 201 (1988).

[10] E. Gutsche, O. Geode. J. Luminesc., 1-2, 200 (1970).

[11] Н.Д. Данилевич. Канд. дис. (М., МЭИ, 2011).

[12] В.А. Теплицкий. Канд. дис. (М, МИЭТ, 1989).

[13] V.S. Zimogorsky, N.A. Yashtulov, V.V. Blinov, N.K. Morozova. Kinetic's Method Determination Oxygen Concentrations with Using Gas Chromatography in $\mathrm{A}^{2} \mathrm{~B}^{6} .30$ IST Conf. Russia, M., MEI (2000) c. 211.

[14] Н.К. Морозова, Н.Д. Данилевич. ФТП, 44 (4), 458 (2010).

[15] N.K. Morozova, N.D. Danilevich, A.A. Kanakhin. Phys. Status Solidi C, 7 (6), 1501 (2010).
[16] N.K. Morozova, D.A. Mideros, N.D. Danilevich. Oxygen in Optic of Compounds II-VI of View of Theory Anticrossing zones (LAP, Saarbrucken Germany, 2013) [in Russian]. http://earchive.tpu.ru/handle/11683/56199

[17] Н.К. Морозова. Sci. Europe, 51-1 (51), 37 (2020).

[18] Н.К. Морозова. Новое в оптике соединений II-VI-O. Новые возможсности оптической диагностики монокристаллических систем с дебектами (LAP LAMBERT Academic Publishing, Рига, Латвия, 2021). N.K. Morozova. New in the optics of II-VI-O compounds. New possibilities of optical diagnostics of singlecrystal systems with defects (LAP LAMBERT Academic Publishing, Riga, Latvia, 2021). https://morebooks.de/ru/ search?utf8 $=\% \mathrm{E} 2 \% 9 \mathrm{C} \% 93 \& \mathrm{q}=978-620-3-84665-2$

[19] Н.К. Морозова. Sci. Europe, 56-1 (56), 21 (2020).

[20] А.А. Канахин. Канд. дис. (М., МЭИ, 2015).

[21] Н.К. Морозова, А.А. Канахин, А.С. Шнитников. ФТП, 50 (7), 865 (2016).

[22] В.И. Олешко. Автореф. докт. дис. (Томск, ТПУ, 2009).

Редактор Г.А. Оганесян

\section{System of localized excitons on oxygen complexes in CdS}

\author{
N.K. Morozova ${ }^{1}$, I.N. Miroshnikova ${ }^{1,2}$ \\ ${ }^{1}$ National Research University \\ „Moscow Power Engineering Institute“, \\ 111250 Moscow, Russia \\ 2 Institute of Nanotechnology of Microelectronics, \\ Russian Academy of Sciences, \\ 119991 Moscow, Russia
}

Abstract Intense CdS luminescence in the blue and green spectral regions is widely used in all areas of optoelectronics. The study of the optical properties of CdS based on the bands anticrossing theory was carried out with the involvement of broader initial data in the analysis results. Depending on the growing conditions, the presence and change in the concentration of oxygen and intrinsic point defects, which determine the composition of the crystals, are taken into account. The concept of the nonuniform distribution of isoelectronic centers in the bulk of $\mathrm{CdS}$ due to their predominant segregation on compensating stacking faults is introduced. To analyze the optical data, we used the capabilities of the method for constructing band models, which. collects extensive and multilateral information about specific samples. A model of a $\mathrm{CdS} \mathrm{O}$ multizone with stacking faults is presented, which determines the spectrum of green edge emission. An explanation is given of the nature of the edge emission of cadmium sulfide as excitons localized on oxygen complexes.

It is shown that the presence of isoelectronic oxygen centers appear itself in the electro-physical properties of crystals. Recommendations are given for the diagnostics of crystals suitable for the creation of luminescent systems or lasers that are stable in operation. 